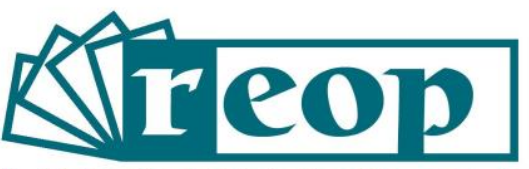

Revista Española de Orientación y Psicopedagogía

\title{
NUEVOS ROLES Y ESTRATEGIAS DE ASESORAMIENTO PSICOPEDAGÓGICO PARA PROMOVER LA EDUCACIÓN INCLUSIVA EN LA COMUNIDAD VALENCIANA ${ }^{1}$
}

\section{NEW ROLES AND STRATEGIES FOR EDUCATIONAL PSYCHOLOGY COUNSELLING TO PROMOTE INCLUSIVE EDUCATION IN THE VALENCIAN COMMUNITY}

\author{
Odet Moliner García ${ }^{2}$ \\ Universitat Jaume I. Facultad de Ciencias Humanas y Sociales. Departamento de Pedagogía, \\ Didáctica de las Ciencias Sociales, la Lengua y la Literatura. Castellón, España \\ Paula Fabregat Mas \\ Universitat Jaume I. Doctoranda en Educación. Castellón, España
}

\section{RESUMEN}

El estudio se sitúa en el ámbito de la orientación educativa y se centra en indagar cómo los asesores psicopedagógicos intervienen en el desarrollo y la formación permanente de los profesionales de la educación para mejorar la educación inclusiva. La nueva organización de la red de Centros de Formación, Innovación y Recursos (CEFIRE) de la Comunidad Valenciana exige repensar los modelos de asesoramiento psicopedagógico y la práctica orientadora desde las funciones otorgadas y las asumidas. Se presentan los resultados de un estudio empírico cuyo objetivo es profundizar en los roles desempeñados y las estrategias utilizadas por los orientadores-asesores para impulsar el avance de la educación inclusiva en los centros educativos. Metodológicamente se adopta un enfoque cualitativo que permite realizar un estudio descriptivo a través de grupos focales en los que participan 22 asesores de educación inclusiva de todos los CEFIREs del territorio. Como resultado se obtiene que acompañar, dinamizar, o gestionar recursos, son algunos de los roles desempeñados y que la creación de redes de recursos, el impulso de las metodologías inclusivas o las visitas pedagógicas se identifican como las estrategias más efectivas. En las conclusiones se destaca la importancia de conjugar diversas

1 Fuente de financiación: Generalitat Valenciana: Proyecto I+D Movilización del conocimiento sobre Educación Inclusiva: Roles y Estrategias de los investigadores/formadores en procesos de mejora escolar desde la InvestigaciónAcción Participativa (AICO/2018/066).

${ }^{2}$ Correspondencia: Odet Moliner García. Correo-e: molgar@uji.es , web: Grupo MEICRI 
estrategias para favorecer la movilización del conocimiento y el avance de las prácticas inclusivas. Finalmente se plantean los retos a los que se enfrentan los asesores para desarrollar un modelo de acompañamiento colaborativo más acorde con el enfoque de la inclusión y con las consiguientes implicaciones en el campo de la orientación y la psicopedagogía.

Palabras clave: Asesoramiento, roles, estrategias, educación inclusiva, modelo colaborativo.

\begin{abstract}
The study is situated in the field of educational counselling and focuses on investigating how educational psychologists and school counsellors are involved in the development and in-service training of educational professionals to improve inclusive education. The new organization of the network of Training, Innovation and Resource Centers (CEFIRE) in the Valencian Community requires rethinking the models of educational psychology counselling and counselling practice from the perspective of the functions provided and those assumed. We present the results of an empirical study that aims to deepen the roles played and the strategies used by counsellorsadvisers to promote the advancement of inclusive education in educational centers. Methodologically, a qualitative approach is adopted that allows a descriptive study to be carried out through focus groups in which 22 inclusive education advisers from all the CEFIRE centers in the territory participate. As a result, some of the roles played include accompanying, energizing and managing resources. The creation of resource networks, the promotion of inclusive methodologies or pedagogical visits are identified as the most effective strategies. The conclusions highlight the importance of combining various strategies to promote the mobilization of knowledge and the advancement of inclusive practices. Finally, the challenges faced by advisers in developing a collaborative accompaniment model more in line with the approach to inclusion and with the consequent implications in the field of counseling and psychopedagogy are presented.
\end{abstract}

Key Words: Advisement / consultation, roles, strategies, inclusive education, collaborative model

\title{
Cómo citar este artículo:
}

Moliner, O. y Fabregat, P. (2021). Nuevos roles y estrategias de asesoramiento psicopedagógico para promover la educación inclusiva en la Comunidad Valenciana. Revista Española de Orientación y Psicopedagogía, 32(1), 59-75. https://doi.org/10.5944/reop.vol.32.num.1.2021.30740

\section{Introducción}

Responder con equidad y justicia a la diversidad del alumnado es uno de los principales retos y obligaciones que ha de afrontar la educación del siglo XXI. Para conseguirlo, es necesaria una educación inclusiva que promueva los cambios pertinentes para que realmente tenga lugar una educación de calidad. La UNESCO (2006) define la inclusión como el proceso de abordar y responder a la diversidad de necesidades de todo el alumnado a través de prácticas inclusivas en 
el aprendizaje, las culturas y las comunidades y reducir la exclusión dentro de la educación. Es definida como una filosofía escolar basada en la democracia y la igualdad, que valora positivamente la diversidad y entiende que todo el alumnado ha de aprender a lo largo de la escolaridad con independencia de sus dificultades o características personales (Stainback y Stainback, 1999). Es también un proceso para aprender a vivir con las diferencias de las personas, que requiere humanización y que persigue garantizar la presencia, participación y éxito de todo el alumnado (Echeita y Ainscow, 2011).

Sin embargo, algunos autores perciben cierto desánimo o sensación de estancamiento en referencia al avance de la educación inclusiva (Echeita, 2017; Núñez, 2019). Los cambios positivos que había experimentado el sistema educativo en las últimas décadas se han visto en cierto modo frenados $y$ no ha tenido lugar el impulso continuado que demanda la educación inclusiva (Verdugo y Rodríguez, 2012). Ante este hecho, a la hora de afrontar el reto que supone desarrollar un sistema educativo inclusivo, la adecuada formación del profesorado es una condición que se vuelve innegociable. Tanto la formación inicial como la permanente se convierten en los dos principales focos de actuación. De la primera son responsables las universidades y de la segunda los centros de formación del profesorado a través de los asesores, que desarrollan la orientación psicopedagógica del profesorado.

La literatura internacional establece que una práctica orientadora, desarrollada desde un enfoque de acompañamiento a centros desde procesos de investigación acción colaborativa, influye notablemente en el desarrollo profesional del profesorado que se muestra más proclive a desarrollar una pedagogía inclusiva en sus aulas (Ainscow et al., 2016; Juma et al., 2017, Rebetez y Ramel, 2019) Así, se plantea la investigación-acción como estrategia general de desarrollo de procesos reflexivos, no sólo con el profesorado, sino con el resto de la comunidad (Messiou, 2019) lo cual abre nuevos espacios para la orientación psicopedagógica en un marco de acción y transformación comunitaria.

En España, el debate acerca de la escuela inclusiva está de actualidad. A pesar de que la normativa internacional garantiza el derecho a una educación inclusiva de todos los niños y niñas y ya se ha creado jurisprudencia en ese sentido, las leyes educativas no se han actualizado. Algunas comunidades autónomas como Cataluña y la Comunidad Valenciana han avanzado en la promulgación de un decreto de inclusión. Concretamente en la Comunidad Valenciana el reciente Decreto 104/2018 sienta las bases para el desarrollo de los principios de equidad y de inclusión en el sistema educativo valenciano. Establece que los sistemas educativos deben dirigir sus esfuerzos hacia la mejora de la calidad y la eficiencia de la educación, conjugando calidad y equidad, cohesión social y participación de la ciudadanía. Hace hincapié en que la educación inclusiva parte de que cada alumna y cada alumno tienen necesidades únicas y de la consideración de la diversidad como un valor positivo que mejora y enriquece el proceso de enseñanza y aprendizaje. Destaca la importancia de la participación de la comunidad educativa, motivo por el cual las aulas deben ser acogedoras y abrirse a otros agentes implicados en el proceso educativo. Confiere a los servicios especializados de orientación y a los centros de formación del profesorado (CEFIREs) las funciones de colaboración con los centros docentes y la facilitación del apoyo necesario para desarrollar, seguir y evaluar las respuestas educativas inclusivas que propone el decreto.

En este contexto cobra especial relevancia la nueva organización de los CEFIREs que, a partir de la Resolución de 5 de agosto de 2016, se ampliaron configurando una extensa red conformada por CEFIREs generales de ámbito territorial, y específicos o temáticos. Entre estos últimos está el CEFIRE Específico de Educación Inclusiva de la Comunidad Valenciana que tiene, entre otras funciones, la de coordinar los ámbitos para que la formación sea coherente en todo el territorio. Aparte, se dota de asesores de educación inclusiva a todos los centros de formación generales. El equipo humano de asesores en educación inclusiva está conformado por profesionales de la orientación educativa, con una experiencia profesional variable, pero noveles todos ellos al ser un perfil de nueva creación. 
Así que tenemos, por una parte, las funciones que han de desempeñar los asesores de los centros de formación, que oficialmente se ocupan de: mejorar la cualificación profesional, la adecuación de los conocimientos, hacer evolucionar los métodos y didácticas específicas del profesorado, promover actividades formativas, efectuar seguimiento y supervisión de actividades en centros y colaborar con las administraciones públicas u otras entidades. Además, teniendo en cuenta la legislación referente a los requisitos con los que debe contar un asesor del CEFIRE, la Resolución de 28 de febrero de 2017 y la Orden 64/2012, de 26 de octubre recogen, entre otras, la capacidad de planificación y dinamización a la hora de facilitar la participación activa de los diferentes agentes implicados, la capacidad de trabajar en equipo, la habilidad para motivar al profesorado en la investigación, así como en su práctica docente o la capacidad de construir conocimiento a partir de la reflexión sobre la práctica educativa, a través de la negociación y acompañamiento, y transmitirlo a los profesionales docentes. Así, estas funciones son, en general, amplias y diversas. Farré (2017) destaca como fundamental el acompañamiento a los responsables educativos del desarrollo del niño, sobre todo dentro de la escuela, pero también fuera de ella.

Por otra parte, la reciente Orden $20 / 2019$, de 30 de abril, que regula la organización de la respuesta educativa para la inclusión del alumnado, atribuye funciones específicas a los asesores de educación inclusiva de los CEFIREs. Concretamente, en el artículo 44 se refiere a los CEFIRE como equipos de apoyo a la inclusión y les otorga la función de asesorar, acompañar y apoyar a los centros docentes en el proceso de transformación hacia la inclusión, en la organización de las medidas de respuesta a la diversidad y en la mejora de la calidad educativa, de forma coordinada y en estrecha colaboración con los equipos educativos. También les encomienda la elaboración de un plan de formación básica en inclusión educativa, así como actuaciones formativas que faciliten la adquisición de competencias generales y específicas para el desarrollo de la escuela inclusiva y la respuesta a las necesidades específicas de apoyo educativo del alumnado.

Llegados a este punto hay que puntualizar, siguiendo a Bolívar (1999), que las funciones y los roles no son lo mismo. Mientras que las funciones son otorgadas, los roles se configuran dinámicamente en un proceso de construcción y negociación, lo cual implica alterar percepciones iniciales o preestablecidas que posibilitan la construcción de un nuevo espacio de identidad profesional. En este sentido, y en relación con el rol que desempeñan los asesores en la promoción de un sistema más inclusivo, Durán y Giné (2017) manifiestan que los asesores han de tener en cuenta el desarrollo profesional en contextos abiertos orientados hacia la inclusión, para de esta manera capacitar al docente y al centro en aras de conseguir una mejora educativa. También Monzón (2011) destaca que, en el acompañamiento a centros para avanzar en la inclusión, el asesor desempeña un rol fundamental a la hora de recoger, coordinar y articular información y necesidades de los centros y de otras redes de servicios y agentes comunitarios para diseñar y desarrollar un plan estratégico de intervención. Considera fundamental la coordinación con distintas entidades con la finalidad de establecer colaboraciones de apoyo al sistema escolar, la participación democrática en el centro y el liderazgo del grupo humano del centro escolar reforzando proyectos de transformación (Monzón, 2011):

Todo esto cuidando a las personas e impulsando la formación y el desarrollo personal y profesional de las personas que participan en el equipo de apoyo; no dando recetas sino buscando conjuntamente estrategias que faciliten un mayor funcionamiento autónomo de los centros e impulsando la creación de comunidades de aprendizaje que faciliten tanto la transformación a nivel de centro como el impacto de la misma en la zona (p.123).

Por otra parte, tenemos en nuestros días bastante conocimiento científico sobre educación inclusiva y cómo impulsar el avance del movimiento de la inclusión en los centros educativos está en el centro del debate en la literatura actual, disponiendo de instrumentos para establecer planes de mejora (Arnaiz y Martínez, 2018). En principio, se considera que son múltiples y variadas las estrategias para hacerlo. Sin embargo, todavía no hemos logrado que las evidencias recogidas empíricamente se transfieran a la práctica, de manera que, según Levin (2011) los saberes 
permanecen en un plano alejado de las personas que en realidad son parte de dichos saberes. Así pues, una preocupación actual en el ámbito de la educación inclusiva es, según Moliner y Ramel (2018) y Moliner et al. (2020), cómo movilizar el conocimiento, es decir, cómo potenciar y aumentar la repercusión de la investigación en las comunidades y en los centros docentes y cómo favorecer el trabajo compartido de investigadores, formadores y prácticos. Los asesores que acompañan y forman al profesorado tienen un papel fundamental a la hora de promover las prácticas reflexivas del profesado para el aprovechamiento y la aplicación del conocimiento que ya tenemos en la investigación y el que se genera en los centros docentes sobre educación inclusiva.

Por eso, en esta investigación nos preguntamos ¿cómo los asesores que se ocupan de la formación y acompañamiento al profesorado movilizan el conocimiento de la investigación en educación inclusiva? Es decir, ¿cómo favorecen que el conocimiento teórico del que disponemos a través de la investigación en educación inclusiva tenga un impacto en las prácticas docentes y cómo favorecen que el conocimiento práctico y la reflexión sobre las prácticas inclusivas alimenten el conocimiento académico?

\section{Método}

La presente investigación se enmarca en un diseño cualitativo de tipo descriptivo que aborda desde una perspectiva fenomenológica el objeto de estudio. Se ha optado por la fenomenología porque se preocupa por determinar el sentido dado a los fenómenos y descubrir el significado y la forma en que las personas describen experiencias sobre un hecho concreto (Bisquerra, 2004).

\section{Muestra}

Se contó con la participación de 22 asesores de educación inclusiva, lo que supone el $70 \%$ del total de asesores en educación inclusiva de la Comunidad Valenciana. De ellos, 17 eran mujeres y 5 hombres con edades comprendidas entre los 30 y los 57 años, contando con una experiencia entre 1 y 4 años en la asesoría.

\section{Instrumentos}

El instrumento de recogida de la información fue una guía de grupo focal que constaba de tres apartados: a) protocolo-instrucciones, b) datos sociodemográficos y c) preguntas abiertas sobre roles y estrategias, como las siguientes:

-Como asesores/as ¿cuáles son los roles que desempeñáis para apoyar la movilización del conocimiento de la investigación en educación inclusiva?

-Como asesores-formadores/as, ¿cuáles son las estrategias que lleváis a cabo para apoyar la movilización del conocimiento de la investigación en educación inclusiva? ¿Cuáles creéis que son las más apropiadas? Si las utilizáis, ¿podéis narrar cómo? 
Nuevos roles y estrategias de asesoramiento psicopedagógico... Odet Moliner y Paula Fabregat

\section{Procedimiento}

La información fue recogida a través de la realización de tres grupos focales. En cada grupo participaron asesores de todas las provincias que conforman el territorio. El Grupo Focal 1 (GF1) estaba compuesto por 7 participantes, el Grupo Focal 2 (GF2) por 9 y el Grupo Focal 3 (GF3) por 6. A partir de un protocolo de actuación, se facilitó el desarrollo simultáneo de los grupos. Previo al inicio de cada grupo focal, de acuerdo con los requerimientos éticos de la investigación, se procedió a la firma del consentimiento informado por parte de los participantes, con los datos sociodemográficos y contextuales, el objetivo de la investigación, el proceso de protección de datos, un acuerdo sobre la grabación en soporte digital y las garantías de anonimización de datos personales mediante la utilización de un pseudónimo. Cada grupo focal tuvo una duración aproximada de una hora.

El análisis de datos se ha llevado a cabo con el apoyo del software ATLAS.ti (The Qualitative Data Analysis \& Research Software). Para ello, se han transcrito literalmente las declaraciones de los participantes y posteriormente se ha realizado un análisis de contenido. Este ha tenido, en primer lugar, un carácter deductivo partiendo de dos temas generales (roles y estrategias) que, al encuentro con los datos, han resultado ser tres (roles, estrategias y retos). A continuación, para concretar y abordar aspectos más específicos, se ha realizado un segundo nivel de análisis de carácter inductivo de cada tema, en el que ha tenido lugar una nueva categorización. La codificación de las unidades de significado se ha realizado asignando a cada una la referencia que indica el grupo focal en el cual tuvo lugar (GF1, GF2 y GF3). Al mismo tiempo, también se ha identificado cada uno de los participantes a través de la siguiente codificación $(11,12,13 \ldots$ hasta I22).

\section{Resultados}

La Figura 1 presenta, en un mapa conceptual elaborado con el software CmapTools, la síntesis de los resultados. A continuación, se presentan los datos en forma de verbatim de las declaraciones de los asesores que giran en torno a la cuestión referida a los roles y a las estrategias. Por último, se recogen las situaciones planteadas como retos y/o propuestas de futuro.

En cuanto a los roles desempeñados por los asesores y asesoras para apoyar la movilización del conocimiento en educación inclusiva, de los datos se desprende que las respuestas fueron variadas, tratando de definir en qué consistía su tarea de asesor/a en relación con sus vivencias y experiencias profesionales. Un primer resultado nos lleva a destacar que los roles explicitados respondían a un patrón múltiple y variable que además era no excluyente, lo cual permitía el desarrollo de varios roles de manera simultánea. Los roles referenciados fueron:

- Gestor de Recursos. Algunas de las declaraciones giraron en torno a la gestión de los recursos disponibles: "Elaboramos recursos, diseñamos formaciones y a la vez somos ponentes" (GF2, I15). También al marco de posibilidades de acciones formativas: "Nuestra intervención se basa en organizar formaciones, en difundir recursos, en buscar personas expertas e intervenir nosotras también como ponentes si conocemos el tema" (GF1, I6). 
- Líder de proyectos. Destacaron asumir el liderazgo de las distintas situaciones y ejercer, en cierto modo, de responsables de todo aquello que tiene que ver con la toma de decisiones y diseño de los planes de actuación sobre inclusión: "Lo que estamos haciendo es liderar proyectos de investigación o de innovación en centros piloto. Ahora mismo nos encontramos realizándolo en tres o cuatro centros" (GF1, I1).

- Dinamizador. Otro de los roles que a menudo identificaron los asesores fue el de dinamizador. Entendían que es fundamental una primera acción que actuara como palanca de dinamización y captara el interés de los agentes implicados: "Nuestra tarea también tiene que ir encaminada a despertar la motivación por emprender nuevos retos. Esto es más fácil si somos capaces de ofrecer unas garantías de éxito" (GF3, 117). Afirmaron que se trata de "(...) intentar motivar y que se desinstalen (el profesorado) de la cultura de la queja, ser críticos e intentar mejorar a través de la movilización y que no se excusen a la hora de llevar a cabo iniciativas" (GF3, I20).

\section{Figura 1}

Roles, estrategias y retos de los asesores para movilizar el conocimiento en educación inclusiva.

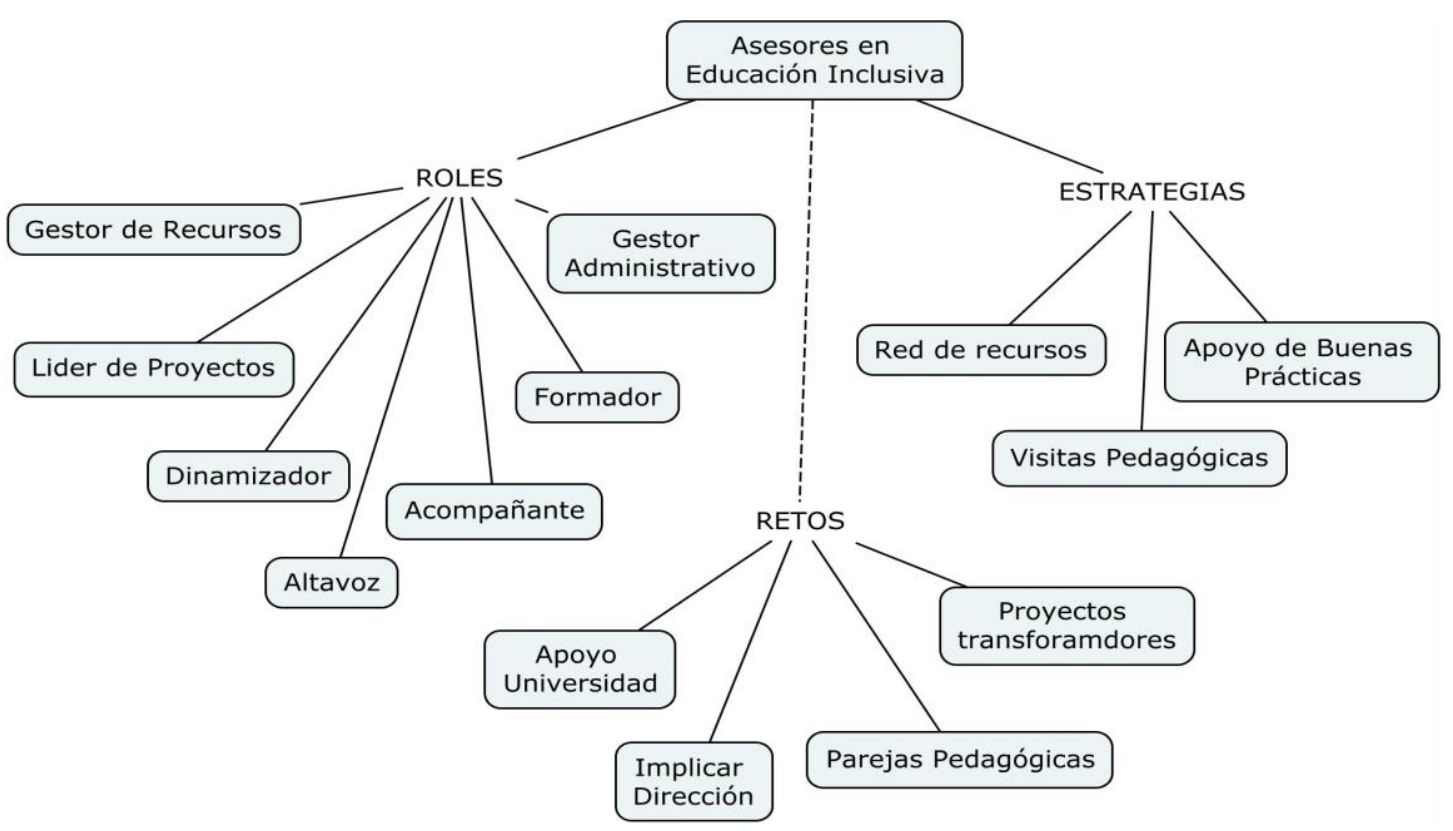

Fuente: Elaboración propia.

- Altavoz. Se hizo hincapié en la importancia de compartir las experiencias de centros, considerando fundamental la transmisión de la información. Este rol lo definieron los propios asesores, de manera didáctica, como altavoz de buenas prácticas. En esta línea, se vuelve crucial potenciar y dar a conocer las buenas prácticas llevadas a cabo:

Otra de las funciones y que bajo mi punto de vista es crucial es la de actuar de lo que yo definiría como altavoz, y así ser el instrumento para visibilizar buenas prácticas realizadas en determinados centros y que otros las puedan conocer e incorporarlas en caso de considerarlo oportuno (GF3, 121).

- Acompañante. Los asesores y asesoras eran conscientes de que todo proceso de cambio requiere un acompañamiento: "A menudo nos piden que los acompañemos, 
que vayamos al claustro, que revisemos el proyecto, es decir, que les hagamos un seguimiento y que les propongamos ideas o formas de actuación" (GF1, I5). Se refirieron a establecer mecanismos de seguimiento que puedan servir de marco de referencia a la hora de posibilitar el desarrollo de las distintas actuaciones inclusivas adaptadas a las necesidades de cada centro:

(...) Es decir, ese acompañamiento más personal y que tiene en cuenta las necesidades más reales, al mismo que se ofrece un feedback que ayude a reflexionar sobre lo que ellos han hecho y que mi experiencia les proporcione posibilidades de mejora (GF3, I20).

- Formador. De manera simultánea a los anteriores, uno de los roles más referenciados fue el de formador: "Nuestro papel consiste en dar herramientas a los docentes. Y un camino para conseguir esto es aumentando las posibilidades de formación. La formación les permite 0 , mejor dicho, nos permite estar actualizados y saber mejor como actuar" (GF3, 120). Los propios asesores y asesoras detectaron que los profesionales de los centros educativos se muestran dispuestos a seguir aprendiendo y contar con más recursos a la hora de atender eficazmente a su alumnado:

Me llama poderosamente la atención y al mismo tiempo me alegra que los centros educativos tengan interés por seguir formándose y estar al día respecto hacia las nuevas metodologías que van apareciendo (...) al igual que muestren inquietud con respecto a la coeducación (GF2, I11).

- Gestor administrativo. Consideraron que facilitar el camino a los profesionales de la educación también es de su incumbencia. Por ejemplo, muchos coincidían en otorgarse un rol de gestor administrativo para ayudar en los farragosos trámites burocráticos y administrativos de los centros:

Tenemos que ser accesibles y ofrecer facilidades. Somos conscientes que tiene que haber una regulación (...) Pero a veces te encuentras con algunas trabas que ralentizan los procesos. Pienso que debemos hacer todo lo posible para agilizar trámites, al menos en la parte que nos toca (GF2, I16).

Asesorar parece una tarea administrativa. Yo, personalmente, paso mucho tiempo delante del ordenador resolviendo cosas administrativas, cuando yo pienso que como asesores deberíamos estar en los centros y muchas veces a los centros puedes ir muy poco porque tienes que estar haciendo otras cuestiones (GF1, I2).

A veces hago un asesoramiento administrativo, muy vinculado a requerimientos de los grupos de trabajo, de lo que tenemos que presentar. En el caso de los seminarios, qué documentación se tiene que presentar, cómo podemos hacer la justificación económica (GF2, I10).

En cuanto a las estrategias para apoyar la movilización del conocimiento de la investigación en educación inclusiva, se identificaron las siguientes estrategias como acciones e iniciativas de apoyo al profesorado:

- Red de recursos. Entre las distintas propuestas se destacó la construcción de una especie de punto de encuentro, sin sede física y accesible para todas y todos en caso de necesitar determinados recursos y que facilitara la movilización del conocimiento:

Lo que hemos hecho no solo es crear redes, sino también redes de recursos. Para hacerlo hemos contado con compañeros y compañeras de la universidad, puntos de búsqueda, servicios específicos. Hemos intentado conectar el mundo de los centros educativos con nosotros ya que somos 
esa palanca intermedia con el mundo universitario. Nosotros intentamos llegar a esa movilización del conocimiento que está en la universidad, hacerlo de una manera más sencilla y posible a los centros, buscando ayuda de compañeros y compañeras conocedores de aspectos fundamentales de la inclusión educativa (GF3, I21).

- Visitas pedagógicas. Estas consisten en visitar otros centros y observar de primera mano su manera de trabajar: "Las visitas pedagógicas dentro y fuera de la comunidad, las que conocemos en el CEFIRE específico, consisten en investigar qué es lo que se está haciendo y qué podemos aportar o presentar, aquí o en los centros, que consideramos positivo" (GF3, 118). Cabe señalar que fueron numerosas las alusiones a esta estrategia y que cuentan con un alto grado de apoyo entre los asesores.

Para fomentar unas buenas prácticas, una de las estrategias que yo creo que más motivan son las visitas pedagógicas. Las visitas pedagógicas, aunque todavía no están muy definidas, (...) se llaman En Ruta y ponen en contacto al profesorado con la realidad de los centros. Yo personalmente pienso que es un acierto, porque yo cuando era coordinadora de formación tenía la referencia del CEFIRE. Yo siempre lo diré: estoy muy agradecida porque he hecho muchas visitas pedagógicas antes, que se podían hacer, y creo que, para mí, como maestra, creo que ha sido una de las modalidades formativas que ha tenido un mayor impacto (GF1, I1).

Nosotros sí que las pudimos hacer en Pascua en Madrid y la verdad es que me han aportado mucho porque son muy enriquecedoras. Ver cómo trabajan en otros lados y esa implicación por parte del profesorado motiva mucho más que una clase magistral (GF3, I19).

Respecto a qué estrategias son más apropiadas, yo creo que las visitas pedagógicas son experiencias que replantean que ese planteamiento teórico que es imposible, que sea posible, y visibilizar las experiencias podría movilizar muchos cambios. (GF3, I17).

- Apoyar buenas prácticas metodológicas. También fueron muchas las ocasiones en las que se refirieron al concepto de buenas prácticas relacionadas con situaciones de enseñanza- aprendizaje inclusivas en el aula. Se referían a distintas estrategias metodológicas de carácter activo y participativo y se situaron como impulsores de estrategias de aprendizaje cooperativo, de aprendizaje servicio u otras de carácter colaborativo:

En mi caso, por ejemplo, existe la voluntad de impulsar el aprendizaje cooperativo con la intención de facilitar una herramienta para favorecer el proceso de enseñanza - aprendizaje. En este sentido cojo como referencia algunas de las iniciativas propuestas por la Universidad de Vic (GF2, I16).

(...) muchas veces hay buenas prácticas en los centros y si las visibilizamos, detectamos y ligamos a procesos de investigación, los docentes cada vez mejoran su actividad y es más innovadora e inclusiva, de alguna manera podemos avanzar en ese proceso. Además, es importante hacer cadena de transmisión para hacer llegar a través de esa formación una transmisión de conocimientos y así la investigación pueda llegar a los centros. Es decir, una correa que facilite y lleve esa formación a los centros (GF3, I21). 
Las parejas pedagógicas son una buena práctica de colaboración: "Formación que hemos comentado antes de parejas pedagógicas de tutores y profesorado de audición y lenguaje" (GF2, I15).

En cuanto a las mejoras, se plantearon como retos:

- Contar con un mayor apoyo del ámbito universitario. Plantearon la necesidad de contar con un organismo como la universidad a la hora de contrastar, evaluar y dar rigurosidad a las iniciativas y propuestas llevadas a cabo: "Es que no tenemos las herramientas suficientes como para ver el impacto que ha tenido una formación en el centro" (GF1, 16). Consideraron que esto dotará a los asesores de mayor confianza y les empoderará para plantear los planes de actuación:

Entonces ahí sí que la Universidad nos podría ayudar, porque es una cuestión mucho más técnica y sí que nos sería de ayuda. Primero, definir las competencias docentes para la inclusión, (...) hay mucho debate también porque sabemos que es una cuestión de actitud y la actitud es muy difícil de cambiar durante la formación, porque no es solamente una cuestión técnica de procedimientos sino de cambiar la actitud docente. A través de la formación estamos viendo que es complejo. Y, por otra parte, definir una serie de variables que podamos medir antes y después de explicar el tratamiento que en ese caso sería la formación (GF2, I13).

- Implicar a los equipos directivos en los procesos de formación: "El equipo directivo tiene que definir, liderar el cambio, tiene que ser el primero en dar el paso" (GF1, I1). En las relaciones que establecen con los centros, mostraron unanimidad en la necesidad de implicar a los equipos directivos en los procesos de formación, sin desmerecer el papel de la figura de coordinador/a de formación en el centro. Ello tiene una clara repercusión en el proyecto educativo del centro con enfoque inclusivo.

Insistimos siempre y procuramos invitar a que asista el equipo directivo del centro para que la formación no solamente recaiga el coordinador de formación. Y bueno, a partir de esas reuniones periódicas, algunas de ellas sí que son a todos los coordinadores del programa de referencia, y también está muy bien porque entre ellos intercambian un poco las formaciones que están haciendo y generan ideas (GF1, 16).

Hasta ahora, muchas veces el contacto que se realiza en los centros está basado en la persona coordinadora de formación o quién coordina esa acción formativa. Hemos empezado a percutir o involucrar a los equipos directivos, el hecho de la formación no es fruto de un curso sino de que de alguna manera la formación tiene que estar vinculada con el proyecto educativo del centro, vincular a equipos directivos, es decir, esa es una de las estrategias que llevamos en marcha para que de alguna manera ese conocimiento se afiance y genere un cambio real (GF3, I21).

- Mejora del trabajo en parejas pedagógicas. En referencia a la estrategia de las parejas pedagógicas, una de las asesoras comentaba lo siguiente:

(...) organizarlo de manera que las personas que han participado en el primer nivel y que han implantado en sus aulas esas estrategias, lo que harán el año que viene es formar un seminario donde seguir compartiendo y seguir investigando como avanzar en ese sentido. Probablemente serán personas que puedan ser ponentes y enseñar y compartir con otros compañeros que empiecen su experiencia. La idea sería pues: las personas que ya han participado en esa primera formación que sean los formadores de nuevas parejas (GF2, I15). 
- Conectar los proyectos formativos con la realidad escolar para transformarla. Un deseo común de todos los participantes era que su trabajo estuviera más cerca de los centros, de las aulas, en definitiva, de la esencia educativa: "Llegar a los centros desde el CEFIRE no es únicamente para la formación, sino que también es llegar a los claustros y proyectos de centro para poder conseguir cambios". (GF3, I18).

\section{Conclusiones y Discusión}

En relación con los roles, de los resultados se desprende que uno con el que más se identifican los asesores y asesoras es el de gestor de recursos formativos. Al mismo tiempo se destacan los roles de líder, señalando la responsabilidad que conlleva estar al frente de proyectos transformadores, y el de dinamizadores proponiendo acciones y motivando a emprenderlas. Marcelo (1997) ya destacó que el asesor debe dinamizar y optimizar los distintos procesos, tanto los de formación colectiva como aquellos que favorecen el aprendizaje autónomo de maestros y profesores. Es fundamental realizar un trabajo previo de motivación para realmente poder conseguir los objetivos planteados. Por otro lado, han sido muchas las voces que han puesto el acento en destacar el rol de acompañante. En esta línea Correa et al. (2015) establecen que el acompañamiento genera mayor seguridad en los docentes, al tiempo que incrementa su participación y compromiso. También Ventura (2008) y Monzón (2011) afirman que hay que entender el asesoramiento como una manera de acompañar a los docentes, trazando nuevas rutas de comunicación y relación con los mismos, lo cual incluye establecer espacios y tiempos para conocer necesidades, inquietudes o problemas. Relacionado con el rol de acompañante se propone el rol de formador, el cual posibilita que los docentes puedan aprender nuevas maneras de interactuar en el aula y fomentar un aprendizaje basado en la indagación y el descubrimiento por parte del alumnado (García et al., 2019).

Sin embargo, frente a estos roles desempeñados surge uno que tensiona al resto, que la mayoría de los asesores desempeña pero que es cuestionado de manera crítica, como es el rol de gestor administrativo. Este rol es entendido como un alejamiento respecto a su verdadera tarea y lo conciben como algo farragoso y complejo. En este sentido, Sepúlveda y Lagomarsino (2007) afirman que las tareas relacionadas con la gestión administrativa conducentes a la mejora de la calidad de la educación a menudo generan un escenario que se caracteriza por el apego y sobrevaloración de los procedimientos administrativos y su cumplimiento normativo.

En cuanto a las estrategias desarrolladas por los asesores y asesoras para apoyar la movilización del conocimiento de la investigación en educación inclusiva, se destaca la importancia de generar una red de recursos que facilite el intercambio entre los distintos asesores. Se ven como un puente que media entre los centros y entre los centros y la universidad. En esta línea autores como Hernández (2005) coinciden en la importancia de identificar recursos y que estos sirvan de enlace entre los miembros de la comunidad educativa, para de esta manera favorecer el contacto entre centros y profesorado y facilitar el intercambio de información y experiencias. Este resultado se relaciona con el rol de altavoz a la hora de visibilizar buenas prácticas y con el de gestor de recursos. Es muy interesante porque concreta la función orientadora en la creación de lazos, estableciendo puentes entre recursos, instituciones y personas. De ahí la necesidad de destacar la idea de red que, según Fernández (2007) y Muijset et al. (2011) constituye un mecanismo de aprendizaje entre escuelas y posibilita compartir 
recursos eficaces para favorecer una educación inclusiva. De los resultados se desprende que otra de las estrategias referenciadas hace hincapié en la necesidad de impulsar buenas prácticas y apoyar el desarrollo de estrategias metodológicas. Esto tiene que ver con una de las funciones de la orientación educativa, enfocada al apoyo psicopedagógico al profesorado, proporcionando métodos y didácticas específicas. En este caso se trata de estrategias ampliamente referenciadas en la literatura como estrategias inclusivas, como el aprendizaje cooperativo, que favorecen la participación de todo el alumnado, provocan mejoras en el compañerismo y en la motivación, favorecen el incremento del rendimiento académico, mejoran el clima de aula y facilitan la inclusión.

Un elemento fundamental para entender los resultados de esta investigación es tener en cuenta que la figura del asesor/a en educación inclusiva, debido a su reciente creación, se encuentra en un proceso de construcción (Moliner y Sánchez, 2019). Su identidad profesional se está configurando y los resultados del presente trabajo muestran las líneas definitorias que los asesores/as se plantean como retos de futuro. A la vista de las propuestas, los retos planteados exigen superar el modelo formativo de la realización de cursos tan arraigado en los Centros de Profesores pues, de acuerdo con Fernández Tílve y Montero (2007), los cursos, a pesar de ser recurrentes, no hacen más que favorecer una formación consumista, individualista y basada en incentivos externos que no ayudan al desarrollo profesional y a la mejora de la práctica.

Así, los resultados apuntan a una serie de mejoras que nos llevan a considerar cuáles son las implicaciones de este estudio para el campo de la orientación educativa. Los asesores participantes pretenden dar rigurosidad a las propuestas formativas con el apoyo de las universidades, implicar a las direcciones de los centros en los proyectos formativos de centro, apoyar el trabajo en parejas pedagógicas y desempeñar su trabajo más cerca de los centros educativos. Estrechar la relación entre los asesores de los centros de formación y la Universidad puede dotar de mayor rigurosidad las actuaciones que realizan en los centros. A su vez, favorecer una mayor implicación por parte de los equipos directivos en la formación en centros y en los proyectos de transformación inclusivos incidirá en el desarrollo de cambios significativos y en la coordinación de todos los agentes implicados.

La primera implicación del estudio es que los asesores plantean un cambio de modelo de orientación psicopedagógica. Por una parte, parece que bastantes de los roles asumidos y de las estrategias utilizadas por los asesores en educación inclusiva (formación, provisión de materiales y recursos, coordinación, dinamización...) se corresponden con un modelo que Nieto (2001) denomina de intervención. Sin embargo, los retos planteados sugieren que los asesores abogan por un modelo más reflexivo, interactivo y conectado con la realidad escolar, planteando roles y estrategias para la movilización del conocimiento de la investigación en educación inclusiva que ayuden a reconstruir la práctica educativa de manera colaborativa. La inquietud de situarse más próximos a la realidad de los centros, intentando averiguar cómo ayudar al profesorado para que sea él mismo quien diagnostique su problema y encuentre su solución, se aproxima al modelo de facilitación descrito por Nieto (2001). Además, si lo que pretenden los asesores es fomentar el aprendizaje mutuo y el apoyo entre profesionales de la educación, la actividad asesora quedará enmarcada en el modelo colaborativo. Este es el modelo que define las relaciones de interdependencia entre los asesores y el profesorado, un modelo de asesoramiento colaborativo que enfoca la tarea de asesor desde el trabajo conjunto con los equipos docentes, facilitando instrumentos para que reflexionen sobre su propia práctica, compartan nuevos recursos y experiencias y los adapten a su contexto, planificando y desarrollando cambios e innovaciones (Newman y Ingraham, 2016).

Los resultados apuntan que los asesores/as construyen su rol de manera dinámica y cambiante, siempre en relación con las estrategias que favorecen la transformación educativa. A su vez, las estrategias que favorecen la interacción y el intercambio de experiencias compartidas permiten que los profesionales se ayuden mutuamente a expresar lo que hacen en el aula y a definir lo que desearían hacer y ello es una palanca fundamental para el cambio hacia la inclusión, de acuerdo con los trabajos de Ainscow et al. (2016). Estos autores apuntan que la investigaciónacción consigue transformar las prácticas en las escuelas, facilitando procesos interpretativos 
grupales, implicando las diferentes perspectivas de docentes y asesores para fomentar la reflexión crítica, el aprendizaje colaborativo y la crítica mutua para el análisis compartido de las propias prácticas. Por tanto, otra de las implicaciones del trabajo para el campo de la orientación educativa es plantear la investigación-acción como estrategia general de investigación que permite desarrollar un proceso compartido de construcción de conocimiento, desarrollo profesional y mejora de la práctica en el aula (Arnaiz et al., 2018; Moliner et al., 2017).

Alentar el trabajo colaborativo entre los profesionales de la educación mediante estrategias de aprendizaje mutuo, como seminarios y comunidades de práctica, permite explorar diferentes formas de orientación y acompañamiento (Rivera et al., 2013). Las parejas pedagógicas, de acuerdo con los resultados del estudio, se constituyen como una estrategia de colaboración. Ello está en consonancia con los resultados del trabajo de Cotrina et al. (2017) que las consideran como un dispositivo que propicia rediseñar los procesos y los contextos educativos dando cabida a otras formas de hacer que tienen que ver con la educación inclusiva como desarrollar propuestas cooperativas, abrir espacios para el diálogo y el intercambio, acompañar procesos, redefinir roles, etc. Esta estrategia se destaca también en el trabajo de Duran et al. (2019) por favorecer la reflexión, la discusión constructiva entre compañeros, compartir recursos y darse apoyo mutuo.

Conectar con la realidad escolar aprovechando los saberes que allí se generan mediante la práctica reflexiva y los procesos participativos (Levin, 2011) pone al profesorado en el centro del cambio. Este debate participativo sobre los elementos clave de los proyectos de inclusión es el motor para el avance y la transformación de centros. Ello implica poner en valor uno de los elementos clave de la función orientadora: el diálogo. En esta línea el trabajo de Roca, Armengol, Huerta, y Onrubia, (2019) considera fundamental el diálogo y el manejo de diferentes recursos discursivos a lo largo de los procesos de asesoramiento colaborativo para la co-construcción entre asesor y docentes de los problemas y las soluciones. Ello tiene unas claras implicaciones para el ejercicio de la práctica orientadora y cobra especial relevancia en el contexto de la educación inclusiva.

Así pues, los resultados del estudio apuntan que la movilización del conocimiento que ya tenemos sobre educación inclusiva y del que se genera en los centros educativos, exige nuevos roles y estrategias por parte de los asesores en inclusión que se enmarcan en un modelo de asesoramiento o acompañamiento colaborativo. Esta idea es acorde con el trabajo de Juma et al. (2017) que incide en la potencialidad de utilizar la investigación- acción colaborativa en el desarrollo profesional de los maestros y de los orientadores para fomentar la pedagogía inclusiva. Sin embargo, los resultados del estudio no inciden en el enfoque comunitario del trabajo colaborativo tal y como plantea la propuesta de Messiou (2019) que apunta que la investigaciónacción colaborativa debe considerar las opiniones de los estudiantes. Este planteamiento nos lleva a pensar que los asesores limitan su tarea orientadora al ámbito del desarrollo profesional docente y olvidan una función orientadora muy relevante y acorde con el enfoque de la inclusión para la transformación de centros: la participación comunitaria.

Entendemos que los resultados presentados suponen, en conjunto, una contribución al ámbito de la orientación y la psicopedagogía. Sin embargo, hay que remarcar, con todo, las limitaciones que se derivan del diseño metodológico utilizado, centrado en el análisis de un ámbito de la función asesora y de un territorio. Por ello será necesario desarrollar nuevos estudios que analicen con mayor profundidad otras situaciones y procesos de asesoramiento, para poder contrastar y confirmar estos resultados. Así pues, estudios posteriores podrán profundizar en aspectos que han quedado abiertos en nuestro trabajo y que son relevantes para la orientación educativa. Por ejemplo, sería interesante identificar las competencias o perfiles profesionales concretos que se corresponden con los roles identificados en este estudio, para ser desarrollados en los procesos de formación. En esta misma línea también será interesante dar continuidad a la investigación ampliando el análisis de este perfil profesional y sus roles a otros organismos y con otros profesionales que desempeñen asesoramiento psicopedagógico vinculado con el desarrollo de la educación inclusiva como son los Equipos de Orientación Educativa o los Servicios Psicopedagógicos Escolares. Un aspecto que podría abordarse con carácter prospectivo sería 
Nuevos roles y estrategias de asesoramiento psicopedagógico... Odet Moliner y Paula Fabregat

profundizar en qué elementos del proceso de investigación- acción colaborativa son más acordes con el modelo inclusivo y con el enfoque socio comunitario y facilitan el desarrollo de una práctica orientadora más inclusiva.

\section{Referencias bibliográficas}

Ainscow, M., Dyson, A., Goldrick, S. y West, M. (2016). Using Collaborative Inquiry to Foster Equity within School Systems: Opportunities and Barriers. School Effectiveness and School Improvement, 27(1), 7-23. http://dx.doi.org/10.1080/09243453.2014.939591

Arnaiz, P., De Haro, R. y Azorín, C.M. (2018). Redes de apoyo y colaboración para la mejora de la educación inclusiva. Profesorado. Revista de Currículum y Formación de Profesorado, 22(2), 29-49. https://doi.org/10.30827/profesorado.v22i2.7713

Arnaiz, P. y Bisquerra, R. (2004). Metodología de la investigación educativa. La Muralla.

Arnaiz, P. y Martínez, M. (2018). Centros educativos que se autoevalúan y reflexionan sobre sus resultados para mejorar la atención a la diversidad. Revista Española de Orientación y Psicopedagogía, 29 (1) 74 - 90. https://doi.org/10.5944/reop.vol.29.num.1.2018.23295

Bisquerra, R. (2004). Metodología de la investigación educativa. La Muralla.

Bolívar, A. (1999). Roles del psicopedagogo en la innovación y desarrollo del currículum. En F. Peñafiel, González, D. y Amezcua, J.A. (Coords.), La intervención psicopedagógica (pp. 176- 200). Grupo Editorial Universitario.

Correa, J., Bedoya, M. y Agudelo, G. (2015). Formación de docentes participantes en el programa de educación inclusiva con calidad en Colombia. Revista Latinoamericana de Educación Inclusiva, 9 (1) 43- 50. http://www.rinace.net/rlei/numeros/vol9-num1/art2.pdf

Cotrina, M. García, M. y Caparrós, E. (2017). Ser dos en el aula: las parejas pedagógicas como estrategia de co-enseñanza inclusiva en una experiencia de formación inicial del profesorado de secundaria. Aula Abierta, 46, 57-64. https://doi.org/10.17811/rifie.46.2.2017.57-64

DECRETO 104/2018, de 27 de julio, del Consell, por el que se desarrollan los principios de equidad y de inclusión en el sistema educativo valenciano.

Durán, D. y Giné, C. (2017). La formación del profesorado para la educación inclusiva: Un proceso de desarrollo profesional y de mejora de los centros para atender la diversidad. Revista Latinoamericana de Educación inclusiva, 5(2), 153-170. http://repositoriocdpd.net:8080/handle/123456789/1913

Duran-Gisbert, D., Flores-Coll, M., Mas-Torelló, O. y Sanahuja-Gavaldà, J.M. (2019). Docencia compartida en la formación inicial del profesorado: potencialidades y dificultades según los estudiantes y los profesores. REIRE Revista d'Innovació i Recerca en Educació, 12(2), 111. https://doi.org/10.1344/reire2019.12.227430

Echeita, G. (2017). Educación inclusiva. Sonrisas y lágrimas. Aula Abierta, 46, 17-24. https://doi.org/10.17811/rifie.46.2017.17-24 
Nuevos roles y estrategias de asesoramiento psicopedagógico... Odet Moliner y Paula Fabregat

Echeita, G. y Ainscow, M. (2011). La educación inclusiva como derecho: marco de referencia y pautas de acción para el desarrollo de una revolución pendiente. Tejuelo: Revista de Didáctica de la Lengua y la Literatura, 12, 26-46. https://doi.org/10.5209/dida.54093

Farré, A. (2017). El asesoramiento psicopedagógico en contextos escolares. Trialba.

Fernández Tílve, M. D. y Montero, L. (2007). Perspectivas de asesores y profesores sobre las modalidades de formación del profesorado. Revista de Investigación Educativa, 25 (2), 367-388. https://doi.org/10.18861/cied.2014.5.20.20

Fernández, M. (2007). Redes para la innovación educativa. Cuadernos de Pedagogía, 374, 26-30. https://dialnet.unirioja.es/servlet/articulo?codigo $=2487576$

García, A. X. C., Pacheco, R. J. P., García, N. I. C. y Torres, Á. F. R. (2019). La formación del profesorado en el proceso de innovación y cambio educativo. Revista científica Olimpia, 16(54), 140-152.

Juma, S., Lehtomäki, E. y Naukkarinen, A. (2017). Scaffolding teachers to foster inclusive pedagogy and presence through collaborative action research. Educational Action Research, 25 (5), 720-736. https://doi.org/10.1080/09650792.2016.1266957

Levin, B. (2011). Mobilising research knowledge in education. London Review of Education, 9(1), $15-26$.

Marcelo, C. (1997). ¿Quién forma al formador? Un estudio sobre las tareas profesionales y necesidades de formación de asesores de Andalucía y Canarias. Revista de Educación, 313, 249-278. http://www.educacionyfp.gob.es/revista-de-educacion/numeros-revistaeducacion/numeros-anteriores/1997/re313/re313-12.html

Messiou, K. (2019). Collaborative action research: facilitating inclusion in schools, Educational Action Research, 27(2), 197-209.https://doi.org/10.1080/09650792.2018.1436081

Moliner García, O., Arnaiz Sánchez, P. y Sanahuja Ribés, A. (2020). Rompiendo la brecha entre teoría y práctica: ¿Qué estrategias utiliza el profesorado universitario para movilizar el conocimiento sobre educación inclusiva? Educación XX1, 23(1), 173-195. https://doi.org/10.5944/educXX1.23753

Moliner, O. y Ramel, S. (2018). Una mirada sobre el trabajo en red del Laboratoire International sur l'Inclusion Scolaire (LISIS): afianzando una cultura colaborativa para la movilización del conocimiento sobre educación inclusiva. Profesorado. Revista de Currículum y Formación de Profesorado, 22(2), 91-109. https://doi.org/10.30827/profesorado.v22i2.7716

Moliner, O., Sales, A., Sanahuja, A. y Benet, A. (2017). La IAP en el marco de la escuela incluida: la mirada sobre el proceso de los actores implicados. Actas del XIV Congreso Internacional de Educación Inclusiva y las XXXIV Jornadas de Universidades y Educación Inclusiva. Oviedo.

Moliner, O. y Sánchez, T. (2019). Reconstruyendo la figura del asesor en educación inclusiva: Estudio de los procesos de movilización del conocimiento en la formación continua del profesorado. Revista de Educación Inclusiva, 12(1), 291-310. https://revistaeducacioninclusiva.es/index.php/REl/article/view/417

Monzón, J. (2011). Asesoramiento hacia la escuela inclusiva y formación en centros. Revista interuniversitaria de formación del profesorado, 70(25,1), 111-126. https://core.ac.uk/download/pdf/41577489.pdf

Muijs, D., Ainscow, M., Chapman, C. y West, M. (2011). Collaboration and Networking in Education. Springer. 
Newman, D. S. y Ingraham, C. L. (2017). Consultee-Centered Consultation: Contemporary Perspectives and a Framework for the Future. Journal of Educational and Psychological Consultation, 27(1), 1-12. https://doi.org/10.1080/10474412.2016.1175307

Nieto Cano, J. M. (2001). Modelos de asesoramiento a organizaciones educativas. En J. Domingo Segovia (coord.) Asesoramiento al centro educativo. Colaboración y cambio en la institución (pp. 147-166), Octaedro.

Núñez Mayán, M. T. (2019). El estancamiento de la inclusión educativa del alumnado con discapacidad. Revisión de su escolarización entre 1985 y 2015. Revista de Educación Inclusiva, 12(1), 67-96. https://revistaeducacioninclusiva.es/index.php/REl/article/view/416

ORDEN 20/2019, de 30 de abril, de la Conselleria de Educación, Investigación, Cultura y Deporte, por la cual se regula la organización de la respuesta educativa para la inclusión del alumnado en los centros docentes sostenidos con fondos públicos del sistema educativo valenciano.

ORDEN 64/2012, de 26 de octubre, de la Conselleria de Educación, Formación y Empleo, por la que se desarrolla el Decreto 231/1997, de 2 de septiembre, por el que se regula la creación, estructura y funcionamiento de los Centros de Formación, Innovación y Recursos Educativos de la Comunitat Valenciana.

Rebetez, F. y Ramel, S. (2019). Collaborer dans une vision inclusive à 360. Educateur, 9, 8-9. http://hdl.handle.net/20.500.12162/3260

RESOLUCIÓN de 28 de febrero de 2017, de la Conselleria de Educación, Investigación, Cultura y Deporte, por la que se convoca concurso de méritos para la selección y nombramiento de asesores y asesoras de formación de centros de formación, innovación y recursos educativos (CEFIRE) de la Comunitat Valenciana. [2017/1717].

Rivera, E. R. E., Flores, K. N. y Santa Cruz, M. J. (2013). El acompañamiento pedagógico: una experiencia en la formación de docentes en servicio en contextos de Educación $\begin{array}{llll}\text { Intercultural } \quad \text { Bilingüe } \quad \text { (EIB). Uni-pluriversidad, } & \text { 13(2), }\end{array}$ https://doi.org/10.15517/revedu.v43i1.28446

Rivero, V. M. H. (2005). Asesorando a centros educativos implicados en procesos de mejora escolar. REICE. Revista Iberoamericana sobre Calidad, Eficacia y Cambio en Educación, 3(1), 494-502. https://www.redalyc.org/pdf/551/55130149.pdf

Roca, B., Armengol, M.A., Huerta, L. y Onrubia, J. (2019). Recursos discursivos del asesor en un proceso de asesoramiento colaborativo para la mejora de las prácticas docentes. Profesorado. Revista de Currículum y Formación de Profesorado, 23(2), 441-461. https://doi.org/10.30827/profesorado.v23i2.9725

Sepúlveda, C. y Lagomarsino, M. (2007). Estilos de gestión en educación municipalizada chilena y formación continua del profesorado. REICE. Revista Iberoamericana sobre Calidad,

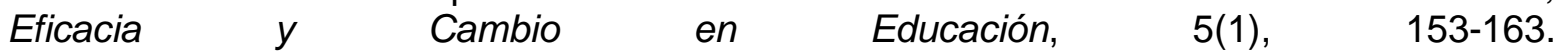
https://revistas.uam.es/reice/article/view/10107

Stainback, S. y Stainback, W. (1999). Aulas inclusivas. La Muralla.

UNESCO (2006). Temario abierto sobre Educación Inclusiva: Materiales de apoyo para responsables de políticas educativas. Santiago, Chile: OREAL/UNESCO (Oficina Regional de Educación de la UNESCO para América Latina y el Caribe).

Ventura, M. (2008). Asesorar es acompañar. Profesorado. Revista de currículum y formación de Profesorado, 12(1), 1-14. https://www.ugr.es/ recfpro/rev121COL2.pdf 
Nuevos roles y estrategias de asesoramiento psicopedagógico... Odet Moliner y Paula Fabregat

Verdugo, M. A. y Rodríguez, A. (2012). La inclusión educativa en España desde la perspectiva de alumnos con discapacidad intelectual, de familias y de profesionales. Revista de Educación, 358, 450-470. https://doi.org/10-4438/1988-592X-RE-2010-358-086

Fecha de entrada: 29 febrero 2020

Fecha de revisión: 28 mayo 2020

Fecha de aceptación: 4 junio 2020 